\title{
Vitesse de Convergence de Certains Estimateurs de Kaplan-Meier de la Régression
}

\author{
Kossi Essona GNEYOU \\ Université de Lomé -Département de Mathématiques BP 1515 Lomé -Togo
}

On considère dans cet article des estimateurs à noyau de la régression basés sur des données censurées à droite qui, dans le cas de non censure, sont identiques aux estimateurs à noyau de la régression de Nadaraya-Watson (1964). La vitesse de convergence uniforme presque sûre sur un intervalle fermé et borné est établie pour ces estimateurs. On utilise ensuite une représentation de la régression par une $V C$ - classe mesurable de fonctions et établit une inégalité exponentielle permettant d'avoir la vitesse de convergence uniforme presque sûre du type Földes et Rejtő (1981) de l'estimateur de Kaplan-Meier de la fonction de répartition conditionnelle.

\section{Introduction}

Soit $X$ l'âge auquel une certaine 'maladie' (infection par le virus du VIH, syntôme, décès, panne ou défaillance etc.) apparait pour la première fois chez un individu. On suppose qu'en examinant un individu, l'observation possible est (i) la maladie est déjà apparue et l'âge exact de la première apparition est connue; (ii) la maladie est déjà apparue mais on ne sait pas précisément à quelle date; (iii) l'individu n'a jamais eu la maladie. Soit $Y$ l'âge de l'individu lors de l'examination. Dans le cas (i), on observe la variable $X$, dans le cas (ii), on sait seulement que $X \leq Y$ et dans le cas (iii), on sait seulement que $X>Y$. Un problème courant en analyse des durées de vie consiste à décrire la loi de probabilité de l'âge aléatoire $X$ d'un individu au cours d'une recherche clinique ou épidémiologique effectuée durant une certaine période d'observation fixée, éventuellement en présence d'un mécanisme de censure (cas (iii)). Parfois le pronostic sur la variable aléatoire $X$ est plus difficile à faire et on cherche une variable intermédiaire $T$ facilement observable et pouvant avoir un caractère prédictif pour l'événement $[X \leq x]$. On exprime ce lien entre $X$ et $T$ par la régression $r(t)=E(X \mid T=t)$. Nous nous intéressons ici à l'estimation non paramétrique de cette régression en considérant pour cela, un modèle de données vérifiant les hypothèses (i), (ii) ou (iii), dites données censurées à droite. Plus précisément soient $\left(X_{1}, T_{1}\right),\left(X_{2}, T_{2}\right), \ldots,\left(X_{n}, T_{n}\right)$ $n$ couples de variables aléatoires réelles i.i.d, de même loi que le couple $(X, T)$ de fonction de répartition (f.r.) $F(x, t)$, de densité $f(x, t)$ et de fonction de répartition conditionnelle (f.r.c.) $F(x \mid t)$ de $X$ sachant $T=t$, toutes les trois inconnues. On note par $f_{0}$ la densité de probabilité de $T$ et par $\Delta_{0}$ le support de $f_{0}$ :

$$
\Delta_{0}=\left\{t \in \mathbb{R} / f_{0}(t)>0\right\}
$$

E-mail address : kossi_gneyou@yahoo.fr 
Soit $Y_{1}, Y_{2}, \ldots, Y_{n}$ une autre suite de v.a.r. i.i.d. de même loi que $Y$, de f.r. $G$, indépendante des $\left(X_{i}, T_{i}\right)$. On suppose que les v.a. $X_{i}, T_{i}$ et $Y_{i}$ sont définies sur un même espace probabilisé $(\Omega, \mathcal{A}, \mathbb{P})$. On considère la régression définie pour tout $t \in \Delta_{0}$ par

$$
\hat{r}(t)=E(\psi(X) \mid T=t)
$$

où $\psi$ appartient à une famille de fonctions numériques mesurables sur $\mathbb{R}$, et le problème de l'estimation non paramétrique de cette régression lorsque les observations disponibles sont de la forme

$$
\left(Z_{1}, \delta_{1}, T_{1}\right), \quad\left(Z_{2}, \delta_{2}, T_{2}\right), \quad \ldots \quad,\left(Z_{n}, \delta_{n}, T_{n}\right)
$$

où pour tout $i=1,2, \ldots, n$,

$$
Z_{i}=\min \left(X_{i}, Y_{i}\right), \quad \delta_{i}=\mathbf{1}_{\left\{X_{i} \leq Y_{i}\right\}}= \begin{cases}1 & \text { si } X_{i} \leq Y_{i} \\ 0 & \text { sinon }\end{cases}
$$

$X_{i}$ est dit censurée à droite par $Y_{i}$ lorsque $\delta_{i}=0$ et le modèle des données est dit sans censure, si $Y=+\infty$ p.s. et donc $G \equiv 0$. Afin d'assurer l'existence presque sûre de $\hat{r}(t)$, nous supposerons dans toute la suite que $E(|\psi(X)|)<+\infty$.

Kaplan et Meier (1958) sont les premiers à considérer le problème de l'estimation non paramétrique de la fonction de répartition d'une v.a. $X$ censurée à droite par une v.a. $Y$ en se basant sur des données de la forme $\left(Z_{i}, \delta_{i}\right), i=1,2, \ldots, n$, dites données censurées à droite. Leurs travaux connaissent par la suite un développement intensif tant en théorie qu'en pratique (voir par exemple ceux de Földes et Rejtő (1981), Földes, Rejtő et Winter (1980 et 1981), Csörgő et Horváth (1983), Gu et Lai (1990), Gneyou (1996 et 1997), Deheuvels et Einmahl (2000), Giné et Guillou (1999), etc.).

Les travaux sur le problème abordé ici sont multiples dans la litérature. Citons par exemple, dans le cas de non censure, ceux de Collomb (1981), Greblicki et al. (1984), Härdle et al.(1988), Stute (1986a,b), Einmahl et Mason (2000), Dersko et Deheuvels (2002), Diakhaby (2002) et dans le cas de données avec censures, ceux de Dabrowska (1987, 1989), Carbonez et al. (1995) et Kohler et Màthé (2002).

Kohler et Màthé (2002) considèrent des estimateurs de la régression basés sur des données censurées à droite en utilisant la méthode de moyenne locale et la méthode des moindres carrés. Ils montrent que l'erreur en moyenne quadratique converge presque sûrement (p.s.) vers 0 quelle que soit la loi du triplet $(X, Y, T)$.

Nous nous proposons ici de développer une méthode d'estimation non paramétrique de la régression $\hat{r}(t)$ basée sur les données censurées $\left(Z_{i}, \delta_{i}, T_{i}\right), i=1,2, \ldots, n$. Nos résultats portent sur la convergence ponctuelle et uniforme des estimateurs obtenus. Le principal résultat conserne la convergence uniforme p.s. de l'estimateur donné au (2.6) avec $\psi$ (resp. $\left.\hat{r}_{n}\right)$ remplacé par $\psi_{s}$ (resp. par $\left.\hat{r}_{n s}\right), s \in J \subset \mathbb{R}$, à une vitesse appropriée. Plus précisément, grâce à une représentation de la régression par une $V C$ - classe mesurable de fonctions ( $V C$ pour Vapnik et Černovenkis), nous montrons que sous des hypothèses convenables et 
raisonnables :

$$
\sup _{s \in J} \sup _{t \in \Delta}\left|\hat{r}_{n s}(t)-\hat{r}_{s}(t)\right|=\mathrm{O}\left(\max \left(\sqrt{\frac{\log \log n}{n h_{n}}}, h_{n}^{\alpha}\right)\right) \quad \text { p.s. }
$$

où $\Delta$ est un intervalle compact de $\mathbb{R}$ et $\alpha$ est l'ordre de la condition de lipschitz qu'on impose à $\varphi_{s}$. La vitesse de convergence que nous obtenons ainsi est similaire à celle obtenue par une approche différente de la notre, par Härdle et al. (1988), dans le cas de données non censurées.

Le travail est organisé comme suite : Dans la partie 2, nous donnons les définitions des estimateurs de la régression pour certaines fonctions $\psi$ et les hypothèses permettant l'existence des estimateurs proposés. Ensuite, nous étudions divers types de convergence dans la partie 3 et présentons les démonstrations des résultats dans la partie $\mathbf{4}$. Une annexe dans laquelle nous rappelons la définition d'une $V C$ - classe de fonctions mesurables est donnée à la fin des démonstrations.

\section{Définition des Estimateurs et Hypothèses}

Ecrivons pour tout $t \in \Delta_{0}, \hat{r}(t)=\frac{\varphi(t)}{f_{0}(t)}$ où

$$
\varphi(t)=\int \psi(x) f(x, t) d x
$$

Pour estimer $\hat{r}(t)$, il suffira donc d'estimer $\varphi(t)$ et $f_{0}(t)$. Soit $K$ un noyau sur $\mathbb{R}$, c'est-à-dire une fonction numérique mesurable vérifiant les conditions suivantes

(K1) $\int K(x) d x=1$;

(K2) Il existe $M \in \mathbb{R}_{+}$telle que $K(x)=0$ pour $|x|>M$;

(K3) $\lim _{|x| \rightarrow+\infty}|x K(x)|=0$,

et soit $\left(h_{n}\right)$ une suite de nombres réels positifs vérifiant

(H1) $h_{n}$ décroit vers 0 lorsque $n \rightarrow+\infty$;

(H2) $n h_{n}$ croit vers $+\infty$ lorsque $n \rightarrow+\infty$;

(H3) $\frac{n h_{n}}{\log n}$ tend vers $+\infty$ lorsque $n \rightarrow+\infty$.

Alors,

- Pour estimer $f_{0}(t)$, nous considérons l'estimateur à noyau $K$ d'une densité de probabilité donné par

$$
f_{n}(t)=\frac{1}{n h_{n}} \sum_{i=1}^{n} K\left(\frac{t-T_{i}}{h_{n}}\right)
$$

(voir par exemple dans Deheuvels (1974))

- Pour estimer $\varphi(t)$, nous considérons l'estimateur $\varphi_{n}(t)$ à noyau $K$ donné par

$$
\varphi_{n}(t)=\frac{1}{h_{n}} \int_{\mathbb{R}^{2}} \psi(x) K\left(\frac{t-s}{h_{n}}\right) d \mu_{n}(x, s)=\frac{1}{n h_{n}} \sum_{i=1}^{n} \frac{\delta_{i} \psi\left(Z_{i}\right)}{1-G_{n}\left(Z_{i}\right)} K\left(\frac{t-T_{i}}{h_{n}}\right),
$$


où $\mu_{n}$ est la mesure empirique associée à l'échantillon $\left(Z_{1}, \delta_{1}, T_{1}\right), \ldots\left(Z_{n}, \delta_{n}, T_{n}\right)$ :

$$
\mu_{n}=\frac{1}{n} \sum_{i=1}^{n} \frac{\delta_{i}}{1-G_{n}\left(Z_{i}\right)} \Delta_{\left\{\left(Z_{i}, T_{i}\right)\right\}}
$$

$\Delta_{a}$ étant la mesure de Dirac en $a$ et $G_{n}$ l'estimateur produit-limite de Kaplan-Meier de la f.r. G donné par (voir e.g. dans Deheuvels et Einmahl (2000))

$$
1-G_{n}(x)=\prod_{\left\{i / Z_{i, n} \leq x\right\}}\left(1-\frac{1-\delta_{i, n}}{n-i+1}\right)
$$

$Z_{1, n} \leq Z_{2, n} \leq \ldots \leq Z_{n, n}$ désignant les $Z_{j}$ ordonnées et $\delta_{i, n}$ les $\delta_{j}$ correspondant à $Z_{i, n}=Z_{j}$, $1 \leq j \leq n$.

Nous obtenons ainsi un estimateur à noyau $K$ de $\hat{r}(t)=E(\psi(X) \mid T=t)$ de la forme

$$
\hat{r}_{n}(t)=\frac{\sum_{i=1}^{n} \frac{\delta_{i} \psi\left(Z_{i}\right)}{1-G_{n}\left(Z_{i}\right)} K\left(\frac{t-T_{i}}{h_{n}}\right)}{\sum_{i=1}^{n} K\left(\frac{t-T_{i}}{h_{n}}\right)} .
$$

Dans le cas général $\psi=\psi_{s}, s \in J$ où $J$ est un intervalle de $\mathbb{R}$, on obtient un estimateur $\hat{r}_{n s}(t)$ de $\hat{r}_{s}(t)=E\left(\psi_{s}(X) \mid T=t\right)$ en remplaçant dans la formule (2.6) ci-dessus, $\hat{r}_{n}$ par $\hat{r}_{n s}$ et $\psi$ par $\psi_{s}$.

\section{Par exemples :}

1) Lorsque $\psi(x)=x$, on obtient un estimateur $r_{n}$ de la régression $r(t)=E(X \mid T=t)$ donné par

$$
r_{n}(t)=\frac{\sum_{i=1}^{n} \frac{\delta_{i} Z_{i}}{1-G_{n}\left(Z_{i}\right)} K\left(\frac{t-T_{i}}{h_{n}}\right)}{\sum_{i=1}^{n} K\left(\frac{t-T_{i}}{h_{n}}\right)} .
$$

2) Lorsque $\psi(x)=\psi_{s}(x)=\mathbf{1}_{\{x \leq s\}}$, on obtient un estimateur $\hat{F}_{n}(x \mid t)$ de la fonction de répartition conditionnelle $F(x \mid t)=\mathbb{P}[X \leq x \mid T=t]$ donné par

$$
\hat{F}_{n}(x \mid t)=\frac{\sum_{i=1}^{n} \frac{\delta_{i}}{1-G_{n}\left(Z_{i}\right)} \mathbf{1}_{\left\{Z_{i} \leq x\right\}} K\left(\frac{t-T_{i}}{h_{n}}\right)}{\sum_{i=1}^{n} K\left(\frac{t-T_{i}}{h_{n}}\right)} .
$$

Dans le cas de non censure, on retrouve l'estimateur de Nadaraya-Watson (1964) de $r(t)$ et celui de la fonction de répartition conditionnelle $F(x \mid t)$ donné par la formule (1.4) dans Einmahl et Mason (2000).

Dans toute la suite, on note par EKM pour estimateur de Kaplan-Meier. Le nom EKM provient de l'estimation de la fonction de répartition $G$ de la censure $Y$ par l'estimateur produit-limite $G_{n}$ de Kaplan et Meier (1958) donné au (2.5) et vérifiant pour tout $T \in \mathbb{R}$ :

$$
\sup _{t \leq T}\left|\frac{1-G_{n}(t)}{1-G(t)}-1\right| \rightarrow 0 \quad \text { p.s. lorsque } n \rightarrow+\infty
$$


(2.9) étant une simple conséquence de la convergence uniforme p.s. de l'EKM $G_{n}$ de $G$ (voir par exemple dans Gu et Lai (1990), Chen et Lo (1997) et les références qui y sont citées).

Dans ce qui suit, nous présentons certains modes de convergence de nos estimateurs. Nous établissons dans le paragraphe 3.1, la convergence ponctuelle sur $\Delta_{0}$ des estimateurs (2.6)-(2.8) pour une classe $\mathcal{F}_{p}$ de fonctions numériques mesurables telle que $\psi \in \mathcal{F}_{p}$ si et seulement si

$$
E\left(\frac{|\psi(X)|^{p}}{(1-G(X))^{p-1}}\right)<+\infty \quad \text { pour tout entier } \quad p \geq 2
$$

Nous établissons ensuite dans le paragraphe 3.2, la convergence uniforme p.s. sur une partie compacte $\Delta \subset \Delta_{0}$ de $\hat{r}_{n}$, pour la classe $\mathcal{F}_{p}$ avec $p=1$ et de l'EKM (2.7) de la régression $r(t)=E(X \mid T=t)$.

Dans le paragraphe $\mathbf{3 . 3}$, nous étudions la convergence uniforme p.s. de $\hat{r}_{n s}$ sur $\Delta$ uniformément en $s \in J$ pour une $V C$-classe $\mathcal{F}$ indexée par $J$. Nous en déduisons un résultat du type Földes et Rejtő (1981) pour l'EKM de la f.r. conditionnelle $F(x \mid t)$ uniformément en $t \in \Delta$. Le dernier paragraphe est consacrée aux démonstrations des résultats.

\section{Convergence des EKM de la régression}

Nous étudions d'abord la convergence ponctuelle et ensuite la convergence uniforme p.s. des estimateurs.

\subsection{Convergence ponctuelle}

Se rappelant de l'expression de $\hat{r}(t)$ en fonction de $f_{0}$ et de $\varphi$ donnée au (2.1), nous faisons les hypothèses suivantes :

(F1) la densité $f_{0}$ de $T$ est continue et strictement positive;

(F2) pour tout $\psi \in \mathcal{F}_{p}(p \geq 1) \hat{r}(t)$ et $v_{p}(t)=E\left(\frac{\psi^{p}(X)}{(1-G(X))^{p-1}} \mid T=t\right)$ sont continues sur le support $\Delta_{0}$ de $f_{0}$.

Alors nous avons

Théorème 3.1 Soit $\psi \in \mathcal{F}_{p}, p \geq 1$. Supposons que les hypothèses (F1), (F2), (K1)-(K3), (H1) et (H2) soient satisfaites. Alors quel que soit $t \in \Delta_{0}$ et quelle que soit la loi du couple $(X, T)$ :

(i) $\hat{r}_{n}(t) \rightarrow \hat{r}(t)$ en probabilité lorsque $n \rightarrow+\infty$

(ii) $\hat{r}_{n}(t) \rightarrow \hat{r}(t)$ en moyenne quadratique lorsque $n \rightarrow+\infty$

La démonstration du Théorème 3.1 est présentée dans la partie 4 . En choisissant $\psi(u)=u$ (resp. $\left.\psi(u)=\mathbf{1}_{\{u \leq x\}}, x \in \mathbb{R}\right)$, on en déduit aisément le corollaire suivant :

Corollaire 3.1 Si $f_{0}, r(t), F(x \mid t)$ et $v_{2}(t)$ sont continues sur $\Delta_{0}$ et si les hypothèses (K1)(K3), (H1) et (H2) sont satisfaites, alors quel que soit $t \in \Delta_{0}$ et quelle que soit la loi du 
couple $(X, T)$ :

(i) $r_{n}(t)$ (resp. $\hat{F}_{n}(x \mid t)$ converge en probabilité vers $r(t)$ (resp. vers $F(x \mid t)$ pour tout $x \in \mathbb{R}$ ) lorsque $n$ tend vers $+\infty$

(ii) $r_{n}(t)$ (resp. $\hat{F}_{n}(x \mid t)$ ) converge en moyenne quadratique vers $r(t)$ (resp. vers $F(x \mid t)$ pour tout $x \in \mathbb{R}$ ) lorsque $n$ tend vers $+\infty$.

En appliquant le Théorème 2 de Greblicki et al. (1984) à l'échantillon $\left(\delta_{i} Z_{i} /\left(1-G\left(Z_{i}\right)\right), T_{i}\right)$, $i=1,2, \ldots, n$, on obtient aussi le résultat suivant englobant la convergence ponctuelle presque sûre de $\hat{r}_{n}(t)$ vers $\hat{r}(t)$ :

Théorème 3.2 Soit $\psi \in \mathcal{F}_{p}$ (avec $p=1$ ). Supposons que les hypothèses (F1), (F2), (K1)(K3) et (H1)-(H3) soient satisfaites avec $K$ minoré par un nombre réel strictement positif. Alors pour tout $t \in \Delta_{0}, \hat{r}_{n}(t) \rightarrow \hat{r}(t)$ p.c. lorsque $n \rightarrow+\infty$.

\subsection{Convergence uniforme p.s. sur un intervalle borné}

Soit $\tau_{F}=\sup \left\{x \in \mathbb{R} / 1-F_{X}(x)=\mathbb{P}[X>x]>0\right\}$ et $\tau_{G}$ defini comme $\tau_{F}$ en remplaçant $F_{X}$ par G. Dans la pratique, $\tau_{F}$ désigne par exemple la date limite des observations de $X$. On supposera ici que $\mathrm{G}$ est continue et que $1-G\left(\tau_{F}\right)=C^{*}>0 \quad$ i.e. $\quad \tau_{F} \leq \tau_{G}$ et considèrera la convergence sur un intervalle compact $\Delta=[a, b] \subseteq \Delta_{0}$.

On dira qu'une fonction numérique $g$ sur $\mathbb{R}$ est uniformément localement lipschitzienne d'ordre $\alpha$ (ulL- $\alpha)$, s'il existe une constante $k_{\alpha}>0$ et un nombre réel $d_{\alpha}<+\infty$ telle que

$$
\sup _{\substack{t, t^{\prime} \in \mathbb{R} \\\left|t-t^{\prime}\right| \leq d_{\alpha}}}\left|g(t)-g\left(t^{\prime}\right)\right| \leq k_{\alpha}\left|t-t^{\prime}\right|^{\alpha}
$$

Pour établir la convergence uniforme p.s. de $\hat{r}_{n}$ vers $\hat{r}$ sur $\Delta$ lorsque $\psi \in \mathcal{F}=\mathcal{F}_{1}$, nous avons besoins des hypothèses suivantes :

(F3) la densité $f_{0}$ de $T$ est continue sur $\Delta$ et vérifie

$$
\inf _{t \in \Delta} f_{0}(t)=m_{0}>0 \text {. }
$$

(F4) $\varphi$ est continue et ulL- $\alpha$ sur $\Delta_{0}, 0<\alpha \leq 1$.

(F5) Il existe $A>0$ tels que pour tout $\psi \in \mathcal{F}$, pour tout $t \in \Delta$ et tout entier $k \geq 2$, $E\left(|\psi(X)-\hat{r}(t)|^{k} \mid T=t\right) \leq k ! A^{k}$.

(K4) $K$ est ulL- $\alpha$ sur $\mathbb{R}, 0<\alpha \leq 1$.

(H4) $\frac{\log \log n}{n}=\mathrm{o}\left(h^{4}\right)$.

Alors nous avons

Théorème 3.3 Soient $\psi \in \mathcal{F}=\mathcal{F}_{1}$ et $\hat{r}_{n}$ associé à un noyau $K$ borné et à une suite réelle positive $\left(h_{n}\right)$ telles que les hypothèses (F3)-(F5), (K1)-(K4) et (H1)-(H4) soient satisfaites. Alors pour $n$ assez grand

$$
\sup _{t \in \Delta}\left|\hat{r}_{n}(t)-\hat{r}(t)\right|=\mathrm{O}\left(\max \left(\sqrt{\frac{\log \log n}{n h_{n}}}, h_{n}^{\alpha}\right)\right) \quad p . s .
$$


La démonstration est donnée dans la partie 4. On en déduit le corollaire suivant

Corollaire 3.2 Soit $r_{n}$ l'EKM de la régression $r$ associé à un noyau $K$ borné et à une suite réelle positive $\left(h_{n}\right)$ telles que les hypothèses (K1)-(K4) et (H1)-(H4) soient satisfaites. Si de plus

(i) la densité $f_{0}$ de $T$ est ulL- $\alpha$ sur $\Delta_{0}, 0<\alpha \leq 1$ et vérifie $\inf _{t \in \Delta} f_{0}(t)=m_{0}>0$

(ii) $\exists A>0 / \forall t \in \Delta, \forall k \in \mathbb{N}, \quad k \geq 2 \quad E\left(|X-r(t)|^{k} \mid T=t\right) \leq k ! A^{k}$.

Alors pour $n$ assez grand

$$
\sup _{t \in \Delta}\left|r_{n}(t)-r(t)\right|=\mathrm{O}\left(\max \left(\sqrt{\frac{\log \log n}{n h_{n}}}, h_{n}^{\alpha}\right)\right) \quad \text { p.s. }
$$

Par exemple le noyau d'Epanechnikov : $K(x)=\frac{3}{4}\left(1-x^{2}\right) \quad|x| \leq 1$, vérifie les hypothèses $(K 1)-(K 4)$.

Dans ce qui suit, nous établissons un résultat global permettant d'obtenir la convergence uniforme p.s. des EKM de la régression traités dans ce paragraphe, en nous dispensant de l'hypothèse peu usuelle (F5) imposée aux moments conditionnels dans le théorème précédent. En particulier, nous obtenons la convergence uniforme p.s. de l'EKM de la f.r.c. $F(x \mid t)$ donné au (2.8).

\subsection{Convergence uniforme p.s. de l'EKM de la f.r. conditionnelle}

Rappelons l'inégalité suivante due à Talagrand (1994) et établie par Giné et Guillou (1999) pour une $V C$-classe mesurable et uniformément bornée de fonctions (leur proposition 2.2) :

Si $\xi_{1}, \xi_{2}, \ldots, \xi_{n}$ sont $n$ v.a.r. i.i.d et si $\mathcal{F}$ est une $V C$-classe mesurable et uniformément bornée de fonctions telles que $U \geq \sup _{f \in \mathcal{F}}\|f\|_{\infty}$ et $\sigma^{2} \geq \sup _{f \in \mathcal{F}} \operatorname{var}\left(f\left(\xi_{1}\right)\right)$ où $\sigma$ et $U$ sont des nombres réels vérifiant $0<\sigma \leq U$, alors il existe des constantes $C$ et $K_{0}$ ne dépendant que des caractéristiques $A$ et $v$ de la $V C-$ classe telle que

$$
\begin{gathered}
\mathbb{P}\left\{\sup _{f \in \mathcal{F}}\left|\sum_{i=1}^{n}\left(f\left(\xi_{i}\right)-E\left(f\left(\xi_{1}\right)\right)\right)\right|>t\right\} \\
\leq K_{0} \exp \left\{-\frac{t}{K_{0} U} \log \left(1+\frac{t U}{K_{0} V_{n}^{2}}\right)\right\} .
\end{gathered}
$$

$\forall t \geq C \sqrt{\log (A U / \sigma)} V_{n} \quad$ où $\quad V_{n}=\sqrt{n} \sigma+U \sqrt{\log (A U / \sigma)}$.

Nous donnons en annexe la définition d'une $V C$ - classe de fonctions mesurables sur un espace mesurable $(\mathbf{S}, \mathcal{S})$ ainsi que le nombre de recouvrements d'un espace métrique $(E, d)$. Pour plus de détails, voir e.g. dans Pollard (1984) ou dans van der Vaart et Wellner (1996).

$$
\text { Soit } \hat{r}_{s}(t)=E\left(\psi_{s}(X) \mid T=t\right)=\frac{\varphi_{s}(t)}{f_{0}(t)}, \quad t \in \Delta_{0} \text { où } \psi_{s}, s \in J \text { appartient à une famille } \mathcal{F}
$$
de fonctions numériques mesurables vérifiant les conditions suivantes : 
(F6) $\forall s<s^{\prime} \in J, \quad 0 \leq \psi_{s}(x) \leq \psi_{s^{\prime}}(x) \quad \forall x \in \mathbb{R}$.

(F7) La famille $\mathcal{F}$ admet une enveloppe mesurable et bornée $\Psi(x)=\sup _{s \in J}\left|\psi_{s}(x)\right|$ telles que $E(\Psi(X)) \leq m_{1}<+\infty$ et $E\left(\Psi^{2}(X)\right) \leq m_{2}<+\infty$.

(F8) Chaque fonction $\varphi_{s}$ est bornée uniformément en $s$ i.e., $\sup _{s \in J} \sup _{t \in \Delta}\left|\varphi_{s}(t)\right|<+\infty$.

(F9) Chaque fonction $\varphi_{s}$ est ulL- $\alpha, 0<\alpha \leq 1$ uniformément en $s$ i.e., il existe $k_{\alpha}>0$ et $d_{\alpha}<+\infty$ tel que $\sup _{s \in J} \sup _{\left\{t, t^{\prime} \in \Delta,\left|t-t^{\prime}\right| \leq d_{\alpha}\right\}}\left|\varphi_{s}(t)-\varphi_{s}\left(t^{\prime}\right)\right| \leq k_{\alpha}\left|t-t^{\prime}\right|^{\alpha}$.

Alors nous avons

Théorème 3.4 Soient $\psi_{s} \in \mathcal{F}$ et $\hat{r}_{n s}$ l'EKM de la régression $\hat{r}_{s}$ associé à un noyau $K$ borné et à une suite réelle positive $\left(h_{n}\right)$ telles que les hypothèses (K1)-(K4), (H1)-(H4) et (F6)-(F9) soient satisfaites. Alors pour $n$ assez grand

$$
\sup _{s \in J} \sup _{t \in \Delta}\left|\hat{r}_{n s}(t)-\hat{r}_{s}(t)\right|=\mathrm{O}\left(\max \left(\sqrt{\frac{\log \log n}{n h_{n}}}, h_{n}^{\alpha}\right)\right) \quad \text { p.s. }
$$

On en déduit le résultat suivant du type Földes et Rejtő(1981) pour $\hat{F}_{n}(x \mid t)$ :

Corollaire 3.3 Soit $\hat{F}_{n}(x \mid t)$ l'EKM de la fonction de répartition conditionnelle $F(x \mid t)$ associé à un noyau $K$ borné et à une suite réelle positive $\left(h_{n}\right)$ telle que les hypothèses (K1)-(K4) et (H1)-(H4) soient satisfaites. Si la densité $f_{0}$ de $T$ est bornée et ulL- $\alpha$ sur $\Delta_{0}, 0<\alpha \leq 1$ alors pour $n$ assez grand

$$
\sup _{x \leq \tau_{F}} \sup _{t \in \Delta}\left|\hat{F}_{n}(x \mid t)-F(x \mid t)\right|=\mathrm{O}\left(\max \left(\sqrt{\frac{\log \log n}{n h_{n}}}, h_{n}^{\alpha}\right)\right) \quad \text { p.s. }
$$

La démonstration du Théorème 3.4 est présentée dans la dernière partie. Celle du Corollaire 3.3 est immédiate. Il suffit d'appliquer le Théorème 3.4 à $\hat{r}_{n s}$ avec $\psi_{x}(y)=\mathbf{1}_{\{y \leq x\}}$ et $\left.J=]-\infty, \tau_{F}\right]$.

\section{Démonstrations des résultats}

\subsection{Preuve du Théorème 3.1}

Nous adaptons le raisonnement de Bosq et Lecoutre (1987). En écrivant

$$
\hat{r}_{n}(t)=\hat{r}(t) \frac{\varphi_{n}(t)}{\varphi(t)} / \frac{f_{n}(t)}{f_{0}(t)}
$$

où $f_{n}$ est l'estimateur à noyau de $f_{0}$ donné au (2.2), nous démontrons d'abord que $\varphi_{n}(t)$ tend vers $\varphi(t)$ en probabilité et en moyenne quadratique lorsque $n$ tend vers $+\infty$ puis, en combinant le théorème de Slutsky et la convergence connue de $f_{n}$ vers $f_{0}$ en probabilité et en moyenne quadratique, nous avons le résultat. 
En tenant compte de (2.9), nous allons travailler avec

$$
\bar{\varphi}_{n}(t)=\frac{1}{n h_{n}} \sum_{i=1}^{n} \frac{\delta_{i} \psi\left(Z_{i}\right)}{1-G\left(Z_{i}\right)} K\left(\frac{t-T_{i}}{h_{n}}\right)
$$

$\bar{\varphi}_{n}$ est égal à $\varphi_{n}$ avec $G_{n}$ remplaçé par $G$. $p \geq 1:$

Posons $U(t)=\frac{\delta_{1} \psi\left(Z_{1}\right)}{1-G\left(Z_{1}\right)} K_{h}\left(t-T_{1}\right)$ où $K_{h}(x)=\frac{1}{h} K\left(\frac{x}{h}\right)$. On a pour tout entier naturel

$$
\begin{aligned}
& E\left(U^{p}(t)\right)=E\left[E\left(U^{p}(t) \mid T\right)\right]=h^{p-1} E\left[K_{h}^{p}\left(t-T_{1}\right) E\left(\left(\delta_{1} \psi\left(Z_{1}\right)\left(1-G\left(Z_{1}\right)\right)^{-1}\right)^{p} \mid T\right)\right] \\
& =h^{p-1} \int K_{h}^{p}(t-s) E\left(\left(\delta_{1} \psi\left(Z_{1}\right)\left(1-G\left(Z_{1}\right)\right)^{-1}\right)^{p} \mid T=s\right) f_{0}(s) d s \\
& =h^{p-1} \int K_{h}^{p}(t-s) \Gamma_{p}(s) d s \\
& =h^{p-1}\left(K_{h}^{p} * \Gamma_{p}\right)(t)
\end{aligned}
$$

avec

$$
\Gamma_{p}(s)=f_{0}(s) E\left(\frac{\psi^{p}(X)}{(1-G(X))^{p-1}} \mid T=s\right)=f_{0}(s) v_{p}(s)
$$

Comme $\Gamma_{p}=f_{0} \cdot v_{p}$ est continue, on applique le lemme de Bochner à $\Gamma_{p}$ et au noyau $\left(\int K^{p}(x) d x\right)^{-1} K^{p}$. On aura

$$
E\left(U^{p}(t)\right)=h^{p-1}\left(K_{h}^{p} * \Gamma_{p}\right)(t) \longrightarrow \Gamma_{p}(t) \int K^{p}(x) d x \quad \forall p \geq 1
$$

lorsque $n \rightarrow+\infty$. En particulier pour $p=1$

$$
E\left(\bar{\varphi}_{n}(t)\right) \longrightarrow \varphi(t)=f_{0}(t) \hat{r}(t)
$$

et pour $p=2$ :

$$
\begin{gathered}
n^{2} \operatorname{var}\left(\bar{\varphi}_{n}(t)\right)=n \operatorname{var}(U(t))=n\left[E\left(U^{2}(t)-(E(U(t)))^{2}\right]\right. \\
\quad=n\left[\frac{1}{h_{n}} h_{n}\left(K_{h_{n}}^{2} * \Gamma_{2}\right)(t)-\left(K_{h_{n}} * \Gamma_{1}\right)^{2}(t)\right]
\end{gathered}
$$

D'où

$$
n h_{n} \operatorname{var}\left(\bar{\varphi}_{n}(t)\right)=h_{n}\left(K_{h_{n}}^{2} * \Gamma_{2}\right)(t)-h_{n}\left(K_{h_{n}} * \Gamma_{1}\right)^{2}(t)
$$

Par suite

$$
n \operatorname{var}\left(\bar{\varphi}_{n}(t) \longrightarrow f_{0}(t) v_{2}(t) \int K^{2}(x) d x\right.
$$

D'après les hypothèses faites dans le théorème, on en déduit que

$$
E\left(\varphi_{n}(t)\right) \longrightarrow \varphi(t) \quad \text { et } \operatorname{var}\left(\varphi_{n}(t)\right) \rightarrow 0
$$

lorsque $n \rightarrow+\infty$ d'où le (i) du Théorème 3.1.

Pour le (ii) on a, compte tenu de (2.9), l'équivalence lorsque $n \rightarrow+\infty$,

$$
E\left|\varphi_{n}(t)-\varphi(t)\right|^{2} \sim E\left|\bar{\varphi}_{n}(t)-\varphi(t)\right|^{2} \leq E\left|\bar{\varphi}_{n}(t)-E \bar{\varphi}_{n}(t)\right|^{2}+\left|E \bar{\varphi}_{n}(t)-\varphi(t)\right|^{2}
$$

et chacun des deux termes à droite de l'inégalité tend vers 0 d'après (4.6) et (4.7) 


\subsection{Preuve du Théorème 3.3}

On écrit

$$
\hat{r}_{n}-\hat{r}=\frac{\varphi_{n}-\varphi}{f_{n}}+\frac{\varphi\left(f_{0}-f_{n}\right)}{f_{0} f_{n}}=\frac{\varphi_{n}-\varphi}{f_{n}}+\hat{r} \frac{\left(f_{0}-f_{n}\right)}{f_{n}}
$$

En notant par $\|f-g\|_{\infty}^{\Delta}=\sup _{t \in \Delta}|f(t)-g(t)|$ pour toutes fonctions bornées $\mathrm{f}, \mathrm{g}$ sur $\Delta$, on a

$$
\left\|\hat{r}_{n}-\hat{r}\right\|_{\infty}^{\Delta} \leq \frac{1}{\left|m_{0}-\left\|f_{0}-f_{n}\right\|_{\infty}^{\Delta}\right|}\left(\left\|\varphi_{n}-\varphi\right\|_{\infty}^{\Delta}+\|\hat{r}\|_{\infty}^{\Delta}\left\|f_{0}-f_{n}\right\|_{\infty}^{\Delta}\right)
$$

Or, pour une loi de $\left(Z_{1}, T_{1}, \delta_{1}=1\right)$ telle que $1-G\left(Z_{1}\right)=\psi\left(Z_{1}\right)$, on a $\varphi_{n}=f_{n}$. Il suffit donc de démontrer que

$$
\left\|\varphi_{n}-\varphi\right\|_{\infty}^{\Delta}=\mathrm{O}\left(\max \left(\sqrt{\frac{\log \log n}{n h_{n}}}, h_{n}^{\alpha}\right)\right) \quad \text { p.s. }
$$

Or on a l'inégalité triangulaire

$$
\left\|\varphi_{n}-\varphi\right\|_{\infty}^{\Delta} \leq\left\|\varphi_{n}-\bar{\varphi}_{n}\right\|_{\infty}^{\Delta}+\left\|\bar{\varphi}_{n}-E \bar{\varphi}_{n}\right\|_{\infty}^{\Delta}+\left\|E \bar{\varphi}_{n}-\varphi\right\|_{\infty}^{\Delta}
$$

L'égalité (4.10) résulte donc de l'inégalité (4.11) et des lemmes suivants :

Lemme 4.1 (i) Si les hypothèses (K1)-(K3) et (F3) sont satisfaites alors pour tout $n$ assez grand on a

$$
\left\|E\left(\bar{\varphi}_{n}\right)-\varphi\right\|_{\infty}^{\Delta}=\mathrm{O}\left(h_{n}^{\alpha}\right)
$$

(ii) Si $\psi \in \mathcal{F}$ et $\hat{r}_{n}$ est associé à un noyau borné, alors pour tout $n$ assez grand

$$
\left\|\varphi_{n}-\bar{\varphi}_{n}\right\|_{\infty}^{\Delta}=\mathrm{O}\left(h_{n}\right) \quad \text { p.s. }
$$

\section{Preuve}

Pour simplifier, on pose $h=h_{n}$. On a pour (i)

$$
E\left(\bar{\varphi}_{n}(t)\right)-\varphi(t)=\left(K_{h} * \varphi\right)(t)-\varphi(t)=\int K(u)[\varphi(t-h u)-\varphi(t)] d u
$$

D'où

$$
\begin{aligned}
\left\|E\left(\bar{\varphi}_{n}\right)-\varphi\right\|_{\infty}^{\Delta} & \leq \sup _{t \in \Delta} \sup _{|u| \leq d_{\alpha}}|\varphi(t-h u)-\varphi(t)| \int|K(u)| d u \\
& +2 \sup _{t \in \Delta}|\varphi(t)| \int_{|u|>d_{\alpha} h^{-1}}|K(u)| d u
\end{aligned}
$$

$\varphi$ étant ulL- $\alpha$ donc uniformément continue sur le compact $\Delta$, on a bien (4.12) compte tenu des hypothèses faites sur le noyau $K$.

Pour (ii) on a

$$
\begin{aligned}
& \left|\varphi_{n}(t)-\bar{\varphi}_{n}(t)\right| \leq \frac{1}{n h} \sum_{i=1}^{n}\left|\delta_{i} \psi\left(Z_{i}\right) K\left(\frac{t-T_{i}}{h}\right)\right|\left|\frac{1}{1-G_{n}\left(Z_{i}\right)}-\frac{1}{1-G\left(Z_{i}\right)}\right| \\
& \leq \frac{\|K\|_{\infty}}{\left(1-G_{n}\left(T_{F}\right)\right)\left(1-G\left(T_{F}\right)\right) h} \sup _{t \in \Delta}\left|G_{n}(t)-G(t)\right| \frac{1}{n} \sum_{i=1}^{n}\left|\psi\left(X_{i}\right)\right|
\end{aligned}
$$


En combinant la Loi Forte des Grands Nombres (LFGN) et la Loi du Logarithme Itéré (LLI) de Földes et Rejtő (1981) (voir (4.28) dans Deheuvels et Einmahl (2000)), on obtient

$$
\left\|\varphi_{n}-\bar{\varphi}_{n}\right\|_{\infty}^{\Delta} \leq \frac{C}{3 h}\left(\frac{\log \log n}{n}\right)^{\frac{1}{2}} \quad \text { p.s. }
$$

où $C=C_{0} \frac{\|K\|_{\infty} E(|\psi(X)|)}{\left(1-G\left(T_{F}\right)\right)^{2}}, C_{0}$ étant une constante positive. D'après l'hypothèse (H4) on a bien (4.13)

Lemme 4.2 Si $\psi \in \mathcal{F}=\mathcal{F}_{\infty}$ et $\hat{r}_{n}$ est associé à un noyau borné et si les hypothèses (K1)(K4), (H1)-(H4) et (F3)-(F5) sont satisfaites alors pour tout $n$ assez grand

$$
\left\|\bar{\varphi}_{n}-E \bar{\varphi}_{n}\right\|_{\infty}^{\Delta}=\mathrm{O}\left(\max \left(\sqrt{\frac{\log \log n}{n h_{n}}}, h_{n}^{\alpha}\right)\right) \quad \text { p.s. }
$$

\section{Preuve}

Posons pour chaque $t \in \Delta$

$$
\bar{\varphi}_{n}(t)-E \bar{\varphi}_{n}(t)=\frac{1}{n h} \sum_{i=1}^{n} W_{i}(t)
$$

où

$$
W_{i}(t)=\frac{\delta_{i} \psi\left(Z_{i}\right)}{1-G\left(Z_{i}\right)} K\left(\frac{t-T_{i}}{h}\right)-E\left(\frac{\delta_{i} \psi\left(Z_{i}\right)}{1-G\left(Z_{i}\right)} K\left(\frac{t-T_{i}}{h}\right)\right)
$$

De la même manière que Bosq et Lecoutre (1987) nous obtenons une majoration de $E(\mid$ $\left.W_{i}^{p}(t) \mid\right) p=1,2, \ldots, n$ de la forme

$$
\begin{aligned}
& E\left(\left|W_{i}^{p}(t)\right|\right) \leq(p-1)\left(\|K\|_{\infty} C_{F} E\left|\psi\left(X_{i}\right)\right|\right)^{p-2}(2 M h) \\
& \quad+M h p ! \sum_{j=2}^{p} \frac{\left(\|K\|_{\infty} C_{F} A\right)^{j-2}}{(p-j) !}\left(\|K\|_{\infty} C_{F} E\left|\psi\left(X_{i}\right)\right|\right)^{p-j}
\end{aligned}
$$

où $C_{F}=\left(1-G\left(T_{F}\right)\right)^{-1}, M$ est un majorant de $K_{h}^{2} * f_{0}$ (en passant par le lemme de Bochner) et $A$ est une constante provenant de la condition (F5). Il existe donc des constantes positives $C_{1}$ et $C_{2}$ telle que

$$
E\left(\left|W_{i}^{p}(t)\right|\right) \leq C_{1}^{p-2} p ! h C_{2} \quad p=2,3, \ldots, \quad i=1, \ldots, n
$$

D'où d'après l'inégalité de Bernstein-Fréchet (voir e.g. Lemme 2.2.11 dans Pollard [26])

$$
\mathbb{P}\left[\frac{1}{n}\left|\sum_{i=1}^{n} W_{i}(t)\right|>z\right] \leq 2 \exp \left(-\frac{n z^{2}}{4 C_{2} h+2 C_{1} z}\right)
$$

On en déduit, compte tenu de la compacité de $\Delta$ que pour tout $\epsilon>0$, il existe des constantes positives $C, D$, et $\beta$ telle que

$$
\mathbb{P}\left[\left\|\bar{\varphi}_{n}-E \bar{\varphi}_{n}\right\|_{\infty}^{\Delta}>\epsilon\right] \leq \frac{C}{h^{\beta}} \exp \left(-D n h \epsilon^{2}\right)
$$

Or si $\epsilon=\mathrm{O}\left(\sqrt{\frac{\log \log n}{n h}}\right)$, le membre de droite de cette dernière inégalité est le terme général d'une série convergente. Le lemme de Borel-Cantelli permet alors de conclure $\square$. 


\subsection{Preuve du Théorème 3.4}

La démonstration du Théorème 3.4 est basée sur le lemme suivant qui lui même est basé sur l'inégalté de Talagrand (3.4)

Lemme 4.3 Sous les hypothèses du Théorème 3.4, on a pour $n$ assez grand

$$
\sup _{s \in J}\left\|\varphi_{n s}-\varphi_{s}\right\|_{\infty}^{\Delta}=\mathrm{O}\left(\max \left(\sqrt{\frac{\log \log n}{n h_{n}}}, h_{n}^{\alpha}\right)\right) \quad p . s .
$$

\section{Preuve}

On a d'abord l'inégalité triangulaire

$$
\begin{aligned}
& \left|\varphi_{n s}(t)-\varphi_{s}(t)\right| \leq\left|\varphi_{n s}(t)-\bar{\varphi}_{n s}(t)\right| \\
& \quad+\left|\bar{\varphi}_{n s}(t)-E \bar{\varphi}_{n s}(t)\right|+\left|E \bar{\varphi}_{n s}(t)-\varphi_{s}(t)\right|
\end{aligned}
$$

En raisonnant comme dans la démonstration du Lemme 4.1 on obtient pour le premier et le troisième terme à droite de l'inégalité ci-dessus

$$
\sup _{s \in J}\left\|\varphi_{n s}-\bar{\varphi}_{n s}\right\|_{\infty}^{\Delta}=\mathrm{O}(h) \quad \text { p.s. }
$$

et

$$
\sup _{s \in J}\left\|E\left(\bar{\varphi}_{n s}\right)-\varphi_{s}\right\|_{\infty}^{\Delta}=\mathrm{O}\left(h_{n}^{\alpha}\right)
$$

Pour le deuxième terme, on a

$$
\begin{gathered}
\left|\varphi_{n s}(t)-E \bar{\varphi}_{n s}(t)\right|=\frac{1}{h} \mid \frac{1}{n} \sum_{i=1}^{n}\left(\frac{\delta_{i} \psi_{s}\left(Z_{i}\right)}{1-G\left(Z_{i}\right)} K\left(\frac{t-T_{i}}{h}\right)-\right. \\
\left.E\left(\frac{\delta_{1} \psi_{s}\left(Z_{1}\right)}{1-G\left(Z_{1}\right)} K\left(\frac{t-T_{1}}{h}\right)\right)\right) \mid \\
\leq \frac{\|K\|_{\infty}}{h}\left|\frac{1}{n} \sum_{i=1}^{n}\left(\frac{\delta_{i} \psi_{s}\left(Z_{i}\right)}{1-G\left(Z_{i}\right)}-E\left(\frac{\delta_{1} \psi_{s}\left(Z_{1}\right)}{1-G\left(Z_{1}\right)}\right)\right)\right|
\end{gathered}
$$

D'où

$$
\begin{aligned}
& \mathbb{P}\left[\sup _{s \in J}\left\|\bar{\varphi}_{n s}-E \bar{\varphi}_{n s}\right\|_{\infty}^{\Delta}>z\right] \\
& \quad \leq \mathbb{P}\left[\left|\frac{1}{n h} \sum_{i=1}^{n}\left(\frac{\delta_{i} \psi_{s}\left(Z_{i}\right)}{1-G\left(Z_{i}\right)}-E\left(\frac{\delta_{1} \psi_{s}\left(Z_{1}\right)}{1-G\left(Z_{1}\right)}\right)\right)\right|>\frac{z}{\|K\|_{\infty}}\right]
\end{aligned}
$$

Posons

$$
\mathcal{G}_{n}=\left\{f_{n s}: \mathbb{R} \times\{0,1\} \rightarrow \mathbb{R}^{+} / \exists \psi_{s} \in \mathcal{F}: f_{n s}(z, \delta)=\frac{\delta \psi_{s}(z)}{n h(1-G(z))}\right\}
$$

D'après le Lemme 3 b) et c) de Giné et Gillou (1999) $\mathcal{G}_{n}$ est une $V C$ - classe mesurable et uniformément bornée par rapport à l'enveloppe $\frac{\Psi}{n h\left(1-G\left(T_{F}\right)\right)}$. 
Soient $\xi_{1}=\left(Z_{1}, \delta_{1}\right), \xi_{2}=\left(Z_{2}, \delta_{2}\right), \ldots, \xi_{n}=\left(Z_{n}, \delta_{n}\right)$.

Les $\xi_{i}$ sont des v.a. i.i.d telles que

$$
\begin{aligned}
& E\left(f_{n s}\left(\xi_{1}\right)\right)=E\left(\frac{\delta_{1} \psi_{s}\left(Z_{1}\right)}{n h\left(1-G\left(Z_{1}\right)\right)}\right) \\
& \quad=E\left(\frac{\psi_{s}\left(X_{1}\right)}{n h\left(1-G\left(X_{1}\right)\right)} E\left(\mathbf{1}_{X_{1} \leq Y_{1}} \mid X_{1}, Y_{1}\right)\right)=E\left(\frac{\psi_{s}\left(X_{1}\right)}{n h} \leq \frac{E(\Psi(X))}{n h} \leq \frac{m_{1}}{n h}\right.
\end{aligned}
$$

De même

$$
\begin{aligned}
& E\left(f_{n s}^{2}\left(\xi_{1}\right)\right)=E\left(\frac{\delta_{1} \psi_{s}\left(Z_{1}\right)}{n h\left(1-G\left(Z_{1}\right)\right)}\right)^{2} \\
& =E\left(\frac{\psi_{s}^{2}\left(X_{1}\right)}{(n h)^{2}\left(1-G\left(X_{1}\right)\right)} \leq \frac{E\left(\Psi^{2}(X)\right.}{(n h)^{2}\left(1-G\left(T_{F}\right)\right)} \leq \frac{C_{F} m_{2}}{(n h)^{2}}\right.
\end{aligned}
$$

avec $C_{F}=\left(1-G\left(T_{F}\right)\right)^{-1}$. D'où

$$
\operatorname{var}\left(f_{n s}\left(\xi_{1}\right)=\frac{1}{n^{2} h^{2}}\left(E\left(\frac{\psi_{s}^{2}\left(X_{1}\right)}{1-G\left(X_{1}\right)}\right)-\left(E\left(\psi_{s}\left(X_{1}\right)\right)^{2}\right) \leq \frac{C_{1}^{2}}{n^{2} h^{2}}=\sigma_{n}^{2}\right.\right.
$$

avec $C_{1}^{2}=m_{1}^{2}+C_{F} m_{2}$. D'autre part on a

$$
\left\|f_{n s}\right\|_{\infty}^{\Delta} \leq \frac{\Psi}{n h\left(1-G\left(T_{F}\right)\right.} \leq \frac{M}{n h\left(1-G\left(T_{F}\right)\right.} \leq \frac{C_{2}}{n h}=U_{n}
$$

avec $C_{2}=M C_{F}$. D'après (4.29) on a

$$
\begin{aligned}
& \mathbb{P}\left[\sup _{s \in J}\left\|\bar{\varphi}_{n s}-E \bar{\varphi}_{n s}\right\|_{\infty}^{\Delta}>z\right] \\
& \quad \leq \mathbb{P}\left[\sup _{s \in J}\left|\sum_{i=1}^{n}\left(f_{n s}\left(\xi_{i}\right)-E\left(f_{n s}\left(\xi_{1}\right)\right)\right)\right|>\frac{z}{\|K\|_{\infty}}\right]
\end{aligned}
$$

Appliquons l'inégalité de Talagrand (3.4) au second membre de (4.30) avec $U_{n}=\frac{C_{2}}{n h}$ et $\sigma_{n}^{2}=\frac{C_{1}^{2}}{n^{2} h^{2}}$. On aura

$$
\begin{aligned}
& \mathbb{P}\left[\sup _{s \in J}\left|\sum_{i=1}^{n}\left(f_{n s}\left(\xi_{i}\right)-E\left(f_{n s}\left(\xi_{1}\right)\right)\right)\right|>C \sqrt{\frac{\log \log n}{n h}}\right] \\
& \quad \leq K_{0} \exp \left\{-\frac{C}{K_{0} C_{2}} \sqrt{n h \log \log n} \log \left(1+\frac{C \cdot C_{2} \sqrt{\log \log n} /(n h)^{3 / 2}}{\left(\frac{C_{1}}{h \sqrt{n}}+\frac{C_{2}}{n h} \sqrt{\log \left(A C_{2} / C_{1}\right)}\right)^{2}}\right)\right\}
\end{aligned}
$$

Or $\frac{n h}{\log n}$ tend vers $+\infty$ lorsque $n$ tend vers $+\infty$. Il en est de même pour $\frac{n h}{\log \log n}$. Donc il existe $N_{0} \in \mathbb{N}^{*}$ tel que l'expression à droite de l'inégalité ci-dessus soit dominée par $K_{0} \exp \{-D \log \log n\}$ pour tout $n \geq N_{0}$, où $D=$ cte $>0$. 
Comme $K_{0} \exp \{-D \log \log n\}$ est le terme général d'une série convergente, le Lemme de Borel-Cantelli combiné avec l'inégalité (4.30) et la majoration ci-dessus permettent d'avoir pour tout $n \geq N_{0}$ :

$$
\sup _{s \in J}\left\|\bar{\varphi}_{n s}-E \bar{\varphi}_{n s}\right\|_{\infty}^{\Delta}=\mathrm{O}\left(\sqrt{\frac{\log \log n}{n h_{n}}}\right) \quad \text { p.s. }
$$

Le Lemme 4.3 découle alors de (4.26), (4.27), (4.31) et de l'inégalité triangulaire (4.25) $\square$.

\section{Preuve du Théorème 3.4}

On raisonne comme dans la démonstration du Théorème 3.3 et on est ramené à établir l'égalité (4.24) d'où le résultat d'après le Lemme $4.3 \square$.

\section{Annexe : Classe de Vapnik et Černovenkis}

Etant donné un espace métrique $(E, d)$ et $\epsilon>0$, le nombre de $\epsilon$-recouvrements de l'espace $(E, d): \mathcal{N}(E, d, \epsilon)$, est défini comme étant le nombre minimal de $d$-boules ouvertes de centres dans $E$ et de rayon $\epsilon$, requis pour couvrir $E$.

Une classe $\mathcal{H}$ de fonctions mesurables sur un espace mesurable $(S, \mathcal{S})$ est une $V C$-classe de fonctions par rapport à l'enveloppe $H$, s'il existe une fonction mesurable $H$ presque partout finie avec $|h| \leq H$ pour tout $h \in \mathcal{H}$, et des nombres réels $A$ et $\nu$ telle que

$$
\mathcal{N}\left(\mathcal{H},\|\cdot\|_{L^{2}(\mathbb{P})}, \epsilon\|H\|_{L^{2}(\mathbb{P})}\right) \leq\left(\frac{A}{\epsilon}\right)^{\nu},
$$

pour tout $\epsilon \in(0,1)$ et toute mesure de probabilité $\mathbb{P} \operatorname{sur}(S, \mathcal{S})$ pour laquelle

$$
\int H^{2} d \mathbb{P}<+\infty
$$

On dira que la classe $\mathcal{H}$ est mesurable si elle peut être paramétrée par un espace métrique complet et séparable $\Theta$ et l'application : $(\theta, x) \mapsto h_{\theta}(x)$ est conjointement mesurable.

Lemme 5.1 (a) Si $\mathcal{H}$ est finie alors $\mathcal{H}$ est une $V C$ - classe par rapport à l'enveloppe $\max \{|h| / h \in \mathcal{H}\}$.

(b) Si $\mathcal{H}=\left\{h_{x}, x \in J\right\}$ où $J$ est une partie de $\mathbb{R}$ et $0 \leq h_{x}(s) \leq h_{y}(s)$ pour tout $x, y \in J$, $x<y$, et $s \in \mathcal{S}$, alors $\mathcal{H}$ est une $V C-$ classe par rapport à $H=\sup \{|h| / h \in \mathcal{H}\}$.

(c) Si $\mathcal{H}_{1}$ et $\mathcal{H}_{2}$ sont des $V C$ - classe par rapport à $\mathcal{H}_{1}$ et à $\mathcal{H}_{2}$ respectivement, alors $\left\{h_{1}+h_{2} / h_{i} \in \mathcal{H}_{i}, i=1,2\right\}$ et $\left\{h_{1}-h_{2} / h_{i} \in \mathcal{H}_{i}, i=1,2\right\}$ sont des $V C$ - classe par rapport $\grave{a}\left(H_{1}^{2}+H_{2}^{2}\right)^{1 / 2}$.

(d) Si $\mathcal{H}$ est une classe de fonctions sur $\left(S^{r}, \mathcal{S}^{r}\right)$ par rapport à une enveloppe $H$ de carré $\mathbb{P}^{r}$-intégrable, et si $\Pi_{m}$ est la $m$ ième projection de Hoeffding par rapport à la mesure de probabilité $\mathbb{P}^{m}$, alors la classe $\left\{\Pi_{m} h / h \in \mathcal{H}\right\}$ est une $V C$-classe par rapport à une enveloppe $K$ telle que $\|K\|_{L^{2}\left(\mathbb{P}^{m}\right)} \leq C_{r}\|H\|_{L^{2}\left(\mathbb{P}^{r}\right)}, 1 \leq m \leq r$, où $C_{r}$ est une constante ne dépendant que de $r$.

\section{Preuve}

Voir dans Giné et Guillou (1999). 


\section{Remerciements}

L'auteur remercie les deux rapporteurs pour leur très grande attention au manuscrit, leurs remarques et leurs suggestions qui ont permis à ce travail d'aboutir.

\section{Références}

[1] Bosq, D. et Lecoutre, J. P. (1987). Théorie de l'estimation fonctionnelle. Economica Paris

[2] Blum, J. R. and Suzarla, V. (1980) Maximal deviation theory of density and failure rate function estimates based on censored data. Multiv. Analysis V. P.R. Krishniah ED. North Holland, New-York 213-222

[3] Carbonez, A., Gyöfi, L. and Van der Meulen, E. C. (1995). Partition-estimates of a regression function under random censoring. Statist. Decisions 13 21-37

[4] Chen, K.and Lo, S.-H. (1997). On the rate of the uniform convergence of the product-limit estimator : Strong and weak laws. Ann. Statist. 25 1050-1087

[5] Collomb, G. (1981). Estimation non paramétrique de la régression : Revue bibliographique. Inst. Statist. Rev. 49 75-93

[6] Csörgő , S.and Horváth, L (1983) The rate of strong uniform consistency for the productlimit estimator. Z. Wahrsch. Verw. Gebiete 61 411-426

[7] Dabrowska, D. M. (1987). Nonparametric regression with censored survival data. Scand. J. Statist. 17 1157-1167

[8] Deheuvels, P. (1974). Conditions nécessaires et suffisantes de convergence presque sûre et uniforme presque sûre des estimateurs de la densité. C.R. Acad. Sci. Paris. Ser. A 278 12171220

[9] Deneuvels, P. and Einmhal, J. H. J. (2000). Functional limit laws for the increments of Kaplan-Meier product-limit processes and applications. Ann. Probab. 3 1301-1335

[10] Derzko, G. and Deheuvels, P. (2002). Estimation non paramétrique de la régression dichotomique - Application biomédicale. C.R. Acad. Sci. Paris Ser. I 334 59-63

[11] Diakhaby, A. (2002). Sur quelques résultats de convergence dans l'inférence d'une classe de processus ponctuels. C.R. Acad. Sci. Paris Ser. I 334 597-602

[12] Einmahl, U. and Mason, D. M. (2000). An empirical processes approach to the uniform consistency of kernel-type function estimators. J. Theoret. Probab. 13 1-37

[13] Földes, A. and ReJtő, L. (1981) A LIL type result for the product-limit estimator. Z. wahrsch verw. Gebiete 56 75-86

[14] Földes, A.,Rejtő, L. and Winter, B. B. (1980) Strong consistency properties of nonparametric estimators for randomly censored data I : the product-limit estimator. Period. Math. Hungar 11 233-250

[15] Földes, A.,Rejtő, L. and Winter, B. B. (1981) Strong consistency properties of nonparametric estimators for randomly censored data II : the estimation of density and failure rate. Period. Math. Hungar 12 15-29

[16] Gneyou, K. E. (1996). Normalité asymptotique d'une fonctionnelle du taux de panne basé sur des données avec censures aléatoires à droite. Ann. Univ. Bénin, sér. Sciences Tome XII, $3-15$ 
[17] Gneyou, K. E. (1997). Functional law of iterated logarithm for a hazard rate process from censored data. J. Rech. Sci. Univ. Bénin (Togo), 1 (2) 44-48

[18] Giné, E. and Guillou, A. (1999). Laws of iterated logarithm for censored data. Ann. Probab. 27 2042-2067

[19] Giné, E. and Guillou, A. (2001). On consistency of kernel density estimators for randomly censored data : rates holding uniformly over adaptive intervals. Ann. I. H. Poincaré-PR 37, 4 503-522

[20] Greblicki, W. Krzyzàl, A. and Pawlak, M(1984). Distribution-free pointwise consistency of kernel regression estimate. Ann. Statist. 12 1570-1575

[21] Gu, M. G. and T. L. (1990) Functional laws of the iterated logarithm for the product-limit estimator of a distribution function under random censorship or truncation. Ann. Probab. 18 160-189

[22] Härdle, W. Janssen, P. and Serfling, R. (1988). Strong uniform consistency rates for estimators of conditional functionals. Ann. Statist. 16 1428-1449

[23] Kaplan, E. K. and Meier, P. (1958) Nonparametric estimation from incomplete observations. J. Amer. Statist. Assoc. 53, 457-481.

[24] Koler, M. and Mathé, K. (2002). Prediction from randomly right censored data. J. Multivariate. Anal. (80) 1 73-100

[25] Nadaraya, E. A. (1964). On estimating regression. Theo. Prob. Appl. 9 141-142

[26] Pollard, D. (1984). Convergence of stochastic processes. Springer Verlag, New-York

[27] Stute, W. (1986a). Conditional empirical processes. Ann. Statist. 14, 638-647

[28] Stute, W. (1986b). On almost sure convergence of conditional empirical distribution functions. Ann. Probab. 14, 891-901

[29] Talagrand, M. (1994). Sharper bounds for gaussian and empirical processes. Ann. Probab. 22 28-76

[30] VAn DeR VAART, A. W. and Wellner, J. A. (1996). Weak convergence and empirical processes with applications to statistics. Springer Verlag, New-York

[31] Watson, G. S. (1964). Smooth regression analysis. Sankhyà A26 359-372 